\title{
EFEITOS DA DESCOERÊNCIA SOBRE CAMINHADAS QUÂNTICAS SOBRE GRAFOS. APLICAÇÕES EM INFORMAÇÃO QUÂNTICA.
}

\author{
Lauro de Jesus Mascarenhas ${ }^{1}$; Fredson Braz Matos dos Santos ${ }^{2}$ \\ 1. Bolsista FAPESB, Graduando em Física, Universidade Estadual de Feira de Santana, e-mail: \\ ljmascarenhas@outlook.com \\ 2. Orientador, Departamento de Física, Universidade Estadual de Feira de Santana, e-mail: braz.uefs@gmail.com
}

PALAVRAS-CHAVE: Descoerência; Algoritmo de Busca; Hipercubo.

\section{INTRODUÇÃO}

Algoritmos baseadas no controle de estados quânticos, ou simplesmente algoritmos quânticos, oferecem um ganho exponencial de eficiência em relação à algoritmos clássicos equivalentes. No entanto, a implementação experimental destes algoritmos esbarra em um grande obstáculo: a descoerência. Resultante da interação do sistema de interesse com o ambiente externo, a descoerência dificulta o controle de estados quânticos e a realização das operações que compõem os algoritmos. A descoerência leva os estados a perderem gradualmente seu caráter quântico e, consequentemente, sua utilidade em computação quântica fica comprometida. Para compreender a descoerência, diversos modelos têm sido propostos. O mais geral requer a análise da interação entre o sistema e o ambiente, mas existem outros modelos de mais fácil implementação, como os modelos de operadores-soma. Neste trabalho, utilizamos o método de operador-soma para estudar descoerência em caminhadas quânticas sobre grafos, buscando compreender os efeitos da descoerência sobre a distribuição de probabilidade e, consequentemente, sobre a eficiência de algoritmos quânticos baseados em caminhadas quânticas (SHENVI, 2003).

Neste trabalho, o objetivo principal é analisar os efeitos da descoerência em caminhadas quânticas sobre grafos. Com este estudo, poderemos analisar diretamente o efeito da descoerência sobre a eficiência de algoritmos quânticos baseados em caminhadas quânticas.

\section{MATERIAL E MÉTODOS OU METODOLOGIA}

O trabalho utilizou investigação da literatura dos métodos utilizados para descrever caminhadas quânticas sobre grafos, efeitos da descoerência em sistemas quânticos e algoritmos de busca baseado em caminhadas quânticas. Além de analisar as distribuições de probabilidades e o tempo de preenchimento para a rede aberta e fechada. Além de aplicar descoerência nos graus de liberdade, interno, externo e em ambos, do caminhante quântico. Em seguida foi feita uma análise de uma caminhada quântica sobre o hipercubo e do algoritmo de busca (SHENVI, 2003). Foi feito também a implementação desse algoritmo com a descoerência e foi feita uma análise de como a eficiência do algoritmo de busca e prejudicada pelo efeito da descoerência.

\section{RESULTADOS E/OU DISCUSSÃO (ou Análise e discussão dos resultados)}

Neste trabalho, foi analisado inicialmente um caminhante movendo sobre uma linha infinita. O sistema é formado por um caminhante quântico que pode realizar $T$ passos em uma rede contendo $N$ sítios. O sentido do movimento é determinando por 
uma "moeda" quântica de duas faces que faz referência ao grau de liberdade interno, isto é, considerando que as faces da moeda sejam descritas pelos estados $|L\rangle$ e $|R\rangle$ o caminhante sofre um deslocamento para a esquerda se a moeda estiver no estado $|L\rangle$ e para a direita caso a moeda esteja no estado $|R\rangle$. Além do grau de liberdade interno, o caminhante quântico possui também o grau de liberdade externo, associado ao estado de posição. Um operador de deslocamento $\hat{D}$ atua no grau de liberdade externo de forma que, a mudança da posição ocorre de acordo com o estado da moeda e segue as relações $\hat{D}|n, L\rangle=|n-1, L\rangle$ e $\hat{D}|n, R\rangle=|n+1, R\rangle$.

O caminhante quântico evolui no tempo de maneira unitária se, e somente se, estiver isolado do ambiente, caso contrário o sistema como um todo sofre a interferência da interação entre o sistema e o ambiente e a evolução deixa de ser unitária. Assim sendo, a descoerência tende a destruir o estado quântico do sistema. Assim sendo, podese representar por $p$ a probabilidade do sistema sofrer um interação com o ambiente e $(1-p)$ a probabilidade do sistema evoluir de forma unitária. A interferência do ambiente pode ser vista na figura 1 .
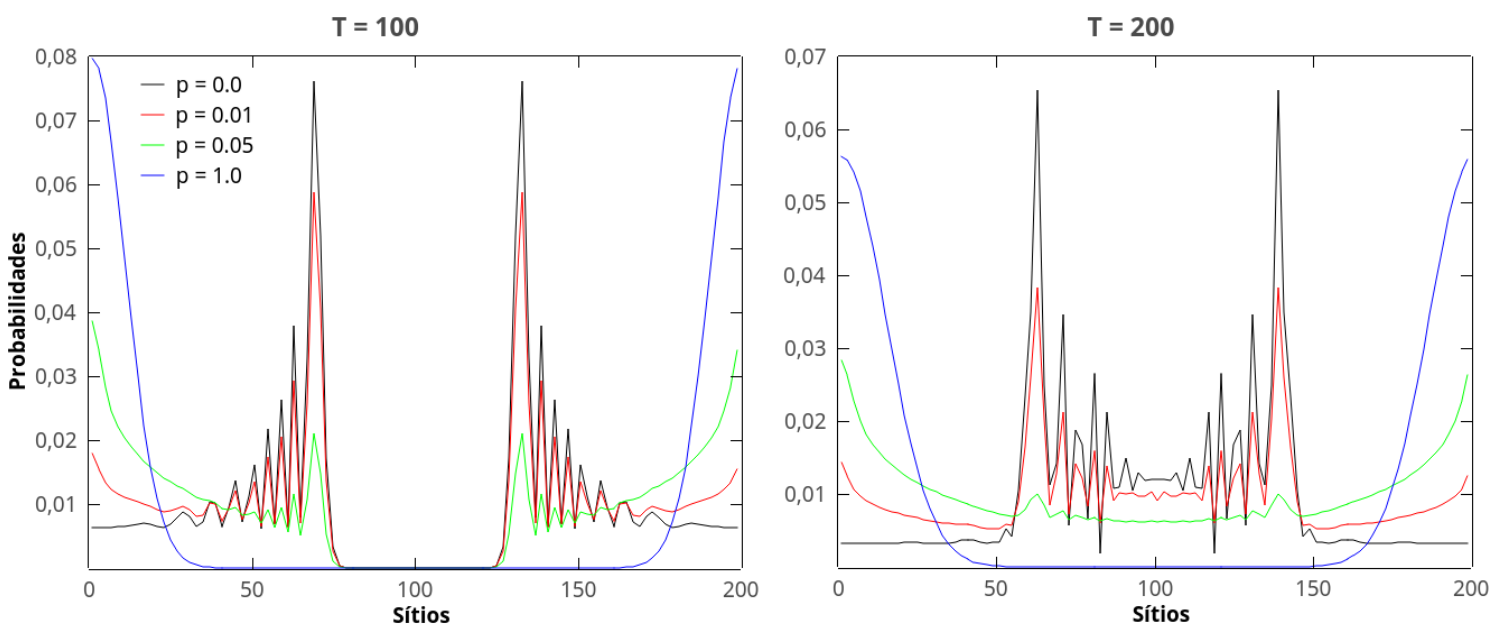

Figura 1: Efeitos da descoerência sobre um caminhante com estado inicial $|\Psi\rangle=\frac{|0\rangle}{\sqrt{2}} \otimes(|L\rangle+i|R\rangle)$, movendo em um grafo unidimensional finito com 200 sítios. Para $p=0$ o sistema é puramente quântico (Evolui de forma unitária), para $p=1$ o estado do caminhante é totalmente afetado pelo ambiente, passando a ter o comportamento de um caminhante clássico.

Este trabalho está focado em redes hipercúbicas pelo fato de já possui um algoritmo de busca bastante eficiente baseado em caminhadas quântica sobre esse tipo de geometria, proposto por Neil Shenvi, Julia Kempe e K. Birgitta Whaley (SHENVI, 2003), algoritmo ficou conhecido como algoritmo SKW (MARQUEZINO, 2010). Para este tipo de rede cada vértice pode ser representado por uma sequência de bits clássicos. Dois vértices são conectados se, e somente se, a diferença entre eles é caracterizada pela inversão de um único bit, ou seja, a distância Hamming é igual a 1. Na figura 2 está representada a distribuições de probabilidades para um caminhante quântico movendose sobre um hipercubo com dimensões $3,5,8$ e 10, e o número de sítios é $2^{n}$. 
$n=3$

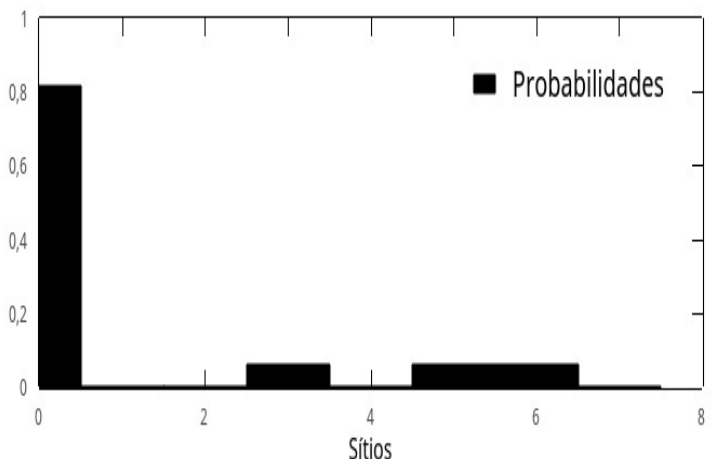

$n=8$

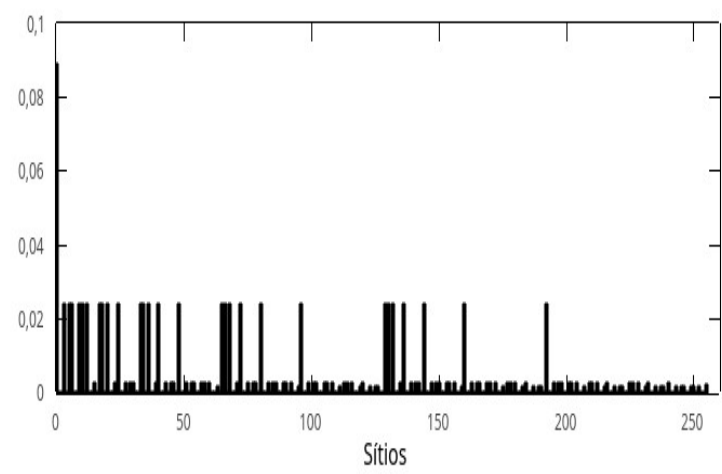

$n=5$

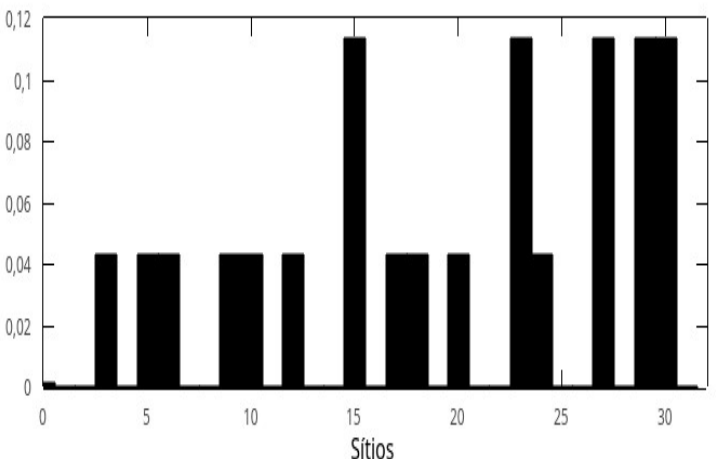

$n=10$

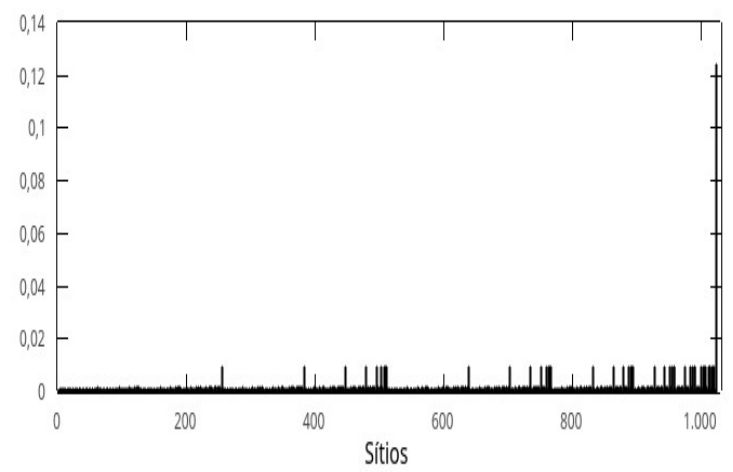

Figura 2: Distribuição de probabilidades para um caminhante quântico movendo-se em um hipercubo para dimensões 3, 5, 8 e 10.

esta forma, é importante analisar como a descoerência afeta a eficiência do algoritmo de busca, a figura 3 mostra como o algoritmo se comporta quando a descoerência atua na posição.
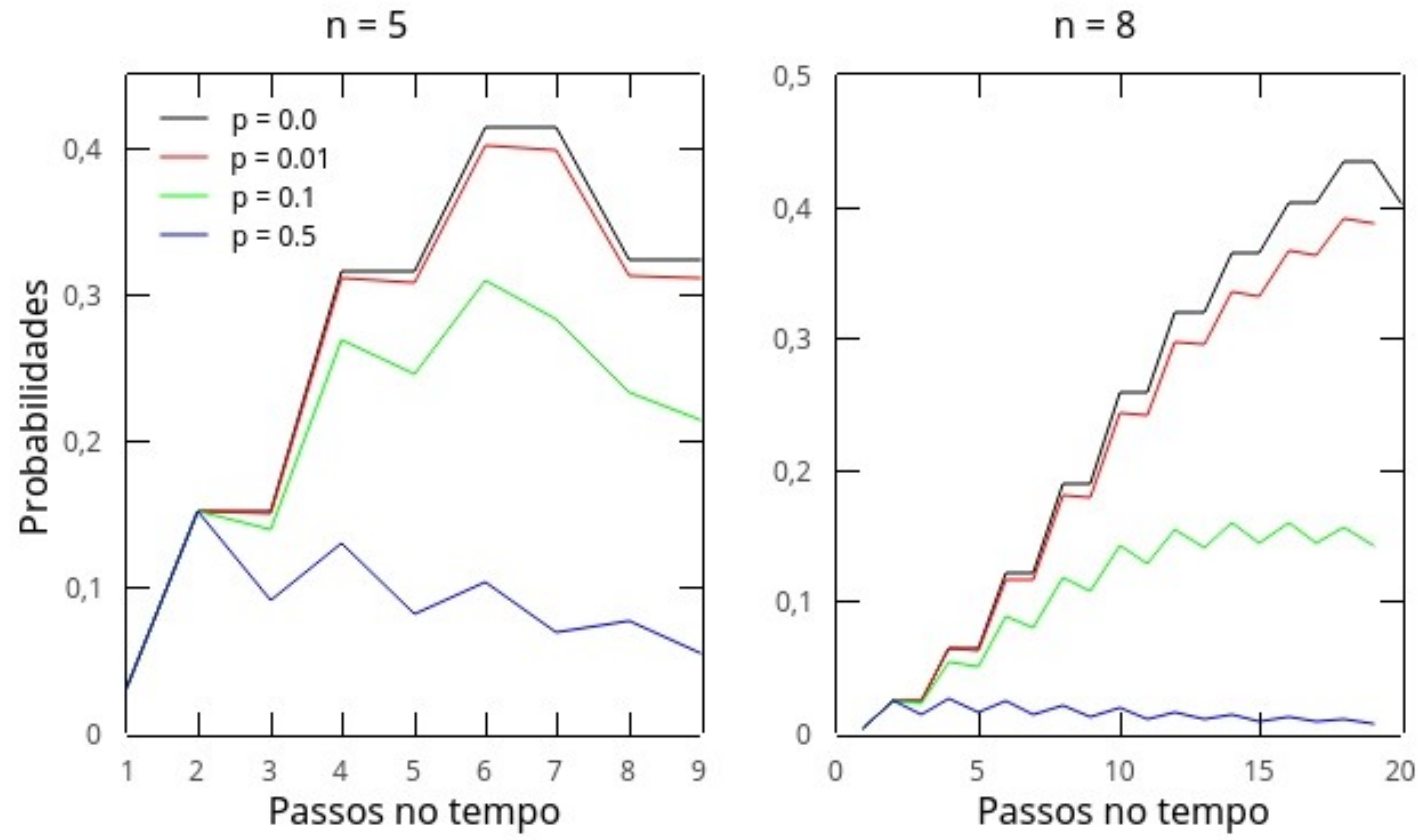

Figura 3: Efeito da descoerência, no grau de liberdade Externo, no algoritmo SKW para redes de dimensões 5 e 8. 
E de maneira mais geral, temos representado na figura 4 a descoerência atuando em ambos os graus de liberdade.
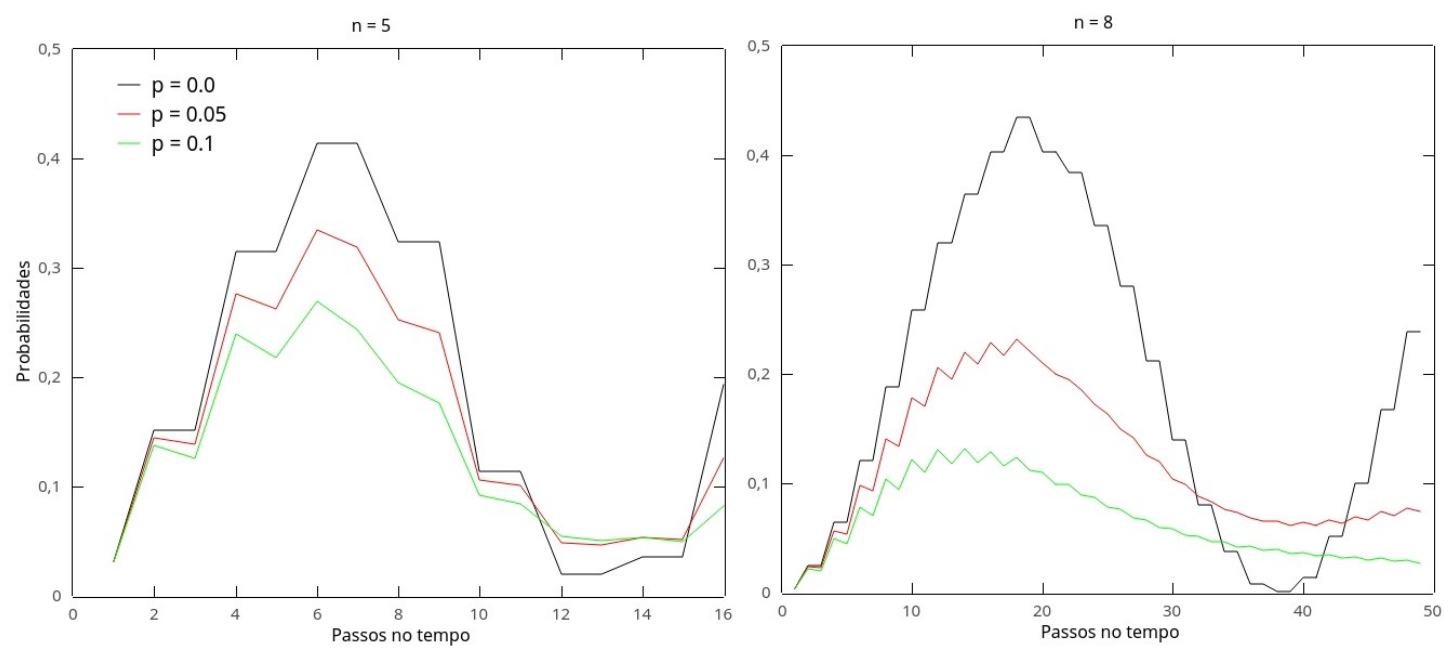

Figura 4: Efeito da descoerência, em ambos os graus de liberdade, no algotimos SKW para uma rede de 5 e 8 dimensões.

\section{CONSIDERAÇÕES FINAIS (ou Conclusão)}

Em relação ao estudo da descoerência nas caminhadas abertas, a distância entre a distribuição de probabilidade e a distribuição estacionária tende a aumentar com a descoerência, mas para pequenos fatores de descoerência, esta distância apresenta redução. Este resultado também foi observado para a caminhada no ciclo apesar da definição de distribuição estacionária ser diferente neste caso. Estes resultados justificam a expectativa que a eficiência de algoritmos podem até aumentar com um pouco de descoerência. Para verificar esta possibilidade, foi feito o estudo do efeito da descoerência sobre o tempo de busca em um algoritmo de busca baseado em caminhadas quântica sobre o hipercubo. A partir dos resultados da caminhada no hipercubo podemos observar que a até determinados valores de $p$ o tempo de busca descrito por Kempe (SHENVI, 2003) continua o mesmo. Entre tanto, a probabilidade de encontrar o estado marco diminui significativamente, para altos valores de $p$, o que influência indiretamente no tempo de busca. Desta forma é possível fazer uma implementações física desde que a interferência do ambiente no sistema seja mínima.

\section{REFERÊNCIAS}

KENDON, VIV. Decoherence in quantum walks-a review. Mathematical Structures in Computer Science, v. 17, n. 6, p. 1169-1220, 2007.

SHENVI, NEIL; KEMPE, JULIA; WHALEY, K. BIRGITTA. Quantum random-walk search algorithm. Physical Review A, v. 67, n. 5, p. 052307, 2003.

MARQUEZINO, FRANKLIN DE L. Análise, simulações e aplicações algorítmicas de caminhadas quânticas. Laboratório Nacional de Computação Científica. Tese doutorado. Petropólis, RJ, 2010.

PORTUGAL, RENATO. Algoritmos quânticos de busca. In: BARCELOS, CÉLIA A. ZORZO, ANDRADE, ELIANA X. L. DE; BOAVENTURA, MAURÍLIO. Notas em matemática aplicada. Sociedade Brasileira de Matemática Aplicada e Computacional. São Carlos, SP, 2010.

GROVER, LOV K. From Schrödinger's equation to the quantum search algorithm. American Journal of Physics, v. 69, n. 7, p. 769-777, 2001. 